\title{
Um Método Unidimensional de Fourier-Gegenbauer para a Resolução da Equação de Helmholtz
}

J.C. OLIVEIRA, Departamento de Matemática, Universidade Federal de Santa Catarina, Cx.P. 476, 88040-900 Florianópolis, SC, Brasil.

J. EYNG, Departamento de Informática e Estatística, Universidade Federal de Santa Catarina, Cx.P. 476, 88040-900 Florianópolis, SC, Brasil.

Resumo. Gottlieb e co-autores propuseram, em [6], um novo método que elimina completamente o fenômeno de Gibbs de expansões em série de Fourier de funções descontínuas, analíticas por partes. O método emprega os coeficientes de Fourier para obter os coeficientes de uma expansão em polinômios de Gegenbauer que representa com acurácia espectral a função dada. Neste trabalho, propomos um método de Fourier-Gegenbauer de resolução numérica de elevada precisão para as equações de Helmholtz unidimensionais. O estudo numérico de casos-teste e comparações com métodos alternativos propostos na literatura evidencia as vantagens da técnica proposta.

\section{Introdução}

Gottlieb e co-autores descreveram, em uma série de papers $[6,7,8,9,10]$, uma metodologia para eliminar o fenômeno de Gibbs presente em expansões em série de Fourier ou Gegenbauer para funções descontínuas, analíticas por partes definidas em $[-1,1]$. No primeiro trabalho da série $([6])$, o assunto central é a remoção do fenômeno da série de Fourier clássica que representa funções analíticas nãoperiódicas.

Vozovoi e co-autores aplicaram este método para resolver problemas a valores no contorno com funções forçantes suaves e não-periódicas ([13]) e equações diferenciais 'stiff' ([12]). No presente trabalho, propomos um método de Fourier-Gegenbauer (FG) adequado para a resolução numérica das equações de Poisson/Helmholtz unidimensionais. O método de Fourier-Gegenbauer que desenvolvemos tem relação direta com aquele abordado no paper seminal de Gottlieb e co-autores [6] (ou seja, Fourier-Galerkin). Este trabalho é organizado da seguinte forma: na Seção 2 , revisamos os resultados principais de Gottlieb e co-autores [6], necessários às seções seguintes. Na Seção 3, desenvolvemos um procedimento de resolução direta das equações unidimensionais de Poisson e Helmholtz, e comparamos os resultados com outros métodos propostos na literatura. Na Seção 4, resolvemos um problema hiperbólico onde o método de Fourier sofre os efeitos do fenômeno de Gibbs. Um sumário das conclusões principais é apresentado na última seção do trabalho. 


\section{O Método de Fourier-Gegenbauer}

Nesta seção, revisamos brevemente os resultados principais de interesse para o presente trabalho.

Seja $I=[-1,1]$ e f uma função arbitrária em $L^{2}(I)$. Seja $\left\{\varphi_{k}\right\}_{k \in \mathcal{N}}$ uma base de polinômios trigonométricos $\varphi_{k}(\mathrm{x}):=\exp (\mathrm{i} \mathrm{k} \pi \mathrm{x})$. Assume-se que os primeiros $2 \mathrm{~N}$ +1 coeficientes de Fourier $c_{k}$, dados por

$$
c_{k}=\frac{1}{2} \int_{I} f(x) \overline{\varphi_{k}(x)} d x
$$

são conhecidos. Definimos

$$
f_{N}(x)=\sum_{k=-N}^{N} c_{k} \varphi_{k}(x)
$$

A idéia principal proposta por [6] é a reconstrução de f, com acurácia espectral na norma do máximo (ou seja, o erro decai a zero mais rapidamente que qualquer potência de $1 / \mathrm{N}$ ), a partir dos coeficientes de Fourier $c_{k}$. Este objetivo é atingido com a expansão em polinômios de Gegenbauer, onde os coeficientes da expansão são obtidos a partir dos coeficientes de Fourier, conforme descrito no teorema a seguir.

$\underline{\text { Definições e Observações: }}$

(i) $C_{l}^{\lambda}(x)$ denota o polinômio de Gegenbauer de ordem 1 .

(ii) $h_{l}^{\lambda}:=\left\|C_{l}^{\lambda}\right\|=\frac{\pi}{2} C_{l}^{\lambda}$ (1) $\frac{\Gamma(\lambda+1 / 2)}{\Gamma(\lambda)(l+\lambda)}, C_{l}^{\lambda}(1)=\frac{\Gamma(l+2 \lambda)}{l ! \Gamma(2 \lambda)}$, onde

$\langle f, g\rangle:=\int_{I}\left(1-x^{2}\right)^{\lambda-1 / 2} f(x) g(x) d x$ e $\|f\|=\langle f, f\rangle^{1 / 2}$.

(iii) $G L=\max _{x \in I}\left|f(x)-\sum_{l=0}^{m} d_{l}^{\lambda} C_{l}^{\lambda}(x)\right|$ onde $d^{\lambda}(l)=\frac{1}{h_{l}^{\lambda}}\left\langle f_{N}, C_{l}^{\lambda}\right\rangle$. GL é o erro entre a função f e a representação em série de Fourier-Gegenbauer de $f_{N}$;

(iv) $R E=\max _{x \in I}\left|f(x)-\sum_{l=0}^{m} \hat{f}^{\lambda}(l) C_{l}^{\lambda}(x)\right|$, onde $\hat{f}^{\lambda}(l)=\frac{1}{h_{l}^{\lambda}}\left\langle f, C_{l}^{\lambda}\right\rangle$. RE é o erro devido ao truncamento da série de Fourier-Gegenbauer (baseado em f com coeficientes exatos);

(v) $T E=\max _{x \in I}\left|\sum_{l=0}^{m}\left(\hat{f}^{\lambda}(l)-d_{l}^{\lambda}\right) C_{l}^{\lambda}(x)\right|$. TE é o erro entre a série de FG truncada com coeficientes exatos (baseados em f) e a série truncada com coeficientes de Fourier-Gegenbauer aproximados (baseados em $f_{N}$ );

(vi) $G L \leq T E+R E$, onde GL é o Erro Global, TE é o Erro de Truncamento e RE representa o Erro de Regularização.

A acurácia espectral é atingida através das condições explicitas no teorema a seguir. Estas condições forçam os erros de truncamento e regularização a decair rapidamente com o número de modos de Fourier.

Teorema 2.1 (Gottlieb, Shu, Solomonoff e Vandeven [6]). Considere uma função analítica não-periódica $f(x)$ definida em I, tal que

$$
\max \left\{\left|\frac{d^{k} f}{d x^{k}}(x)\right|, x \in I\right\} \leq C(\rho) \frac{k !}{\rho^{k}}, \rho \geq 1 .
$$


Assume-se que os coeficientes de Fourier dados pela equação (2.1) são conhecidos para $-N \leq k \leq N$. Sejam $d_{l}^{\lambda}(0 \leq l \leq m)$ os coeficientes da expansão em polinômios de Gegenbauer de $f_{N}(x)$, os quais podem ser expressos explicitamente em termos dos coeficientes de Fourier $c_{k}$ como

$$
d_{l}^{\lambda}=\delta_{l 0} c_{0}+\Gamma(\lambda) i^{l}(l+\lambda) \sum_{0<|k| \leq M} J_{l+\lambda}(\pi k)\left(\frac{2}{\pi k}\right)^{\lambda} c_{k} .
$$

Se $\lambda=\alpha N, m=\beta N$ e $\lambda=\gamma m, \operatorname{com} \alpha, \beta>0$, então

$$
\max \left\{\left|f(x)-\sum_{l=0}^{m} d_{l}^{\lambda} C_{l}^{\lambda}(x)\right|, x \in I\right\} \leq A N^{2} q_{T E}^{N}+A^{\star} q_{R E}^{\beta N},
$$

onde $q_{T E}:=\frac{(\beta+2 \alpha)^{\beta+2 \alpha}}{(2 \pi e)^{\alpha} \alpha^{\alpha} \beta^{\beta}}, q_{R E}:=\frac{(1+2 \gamma)^{1+2 \gamma}}{\rho 2^{1+2 \gamma} \gamma^{\gamma}(1+\gamma)^{1+\gamma}}$ e $\rho(\geq 1)$ denota a distância do intervalo I à singularidade mais próxima de $f(x)$ no plano complexo. $A$ e $A^{\star}$ são constantes.

Sob as condições do teorema, se $\alpha$ e $\beta$ são escolhidos de modo que $q_{T E}<1\left(q_{R E}\right.$ é sempre menor que um, estritamente crescente e tende a $1 / \rho$ quando $\gamma \rightarrow \infty$ ), então os erros de truncamento e regularização são exponencialmente pequenos, ou seja, o erro total é exponencialmente pequeno. Portanto, pode-se calcular os coeficientes de Gegenbauer $\mathrm{d}_{l}^{\lambda}$ da equação (2.4) e aproximar espectralmente $\mathrm{f}(\mathrm{x})$ sobre I, através da expansão

$$
G_{m}^{\lambda}(x):=\sum_{l=0}^{m} d_{l}^{\lambda} C_{l}^{\lambda}(x) .
$$

Na Figura 1, ilustramos o fenômeno com o mesmo exemplo utilizado por Gottlieb, Shu, Solomonoff e Vandeven em [6]: aproximar $f(x)=x$ no intervalo [-1, 1]. A aproximação por série de Fourier apresenta oscilações espúrias próximo aos contornos. Estas oscilações, associadas ao fenômeno de Gibbs, não estão presentes na aproximação produzida pelo método de Fourier-Gegenbauer.

\section{Problemas a Valores no Contorno}

O teorema descrito na seção anterior teve forte influência em áreas onde a presença do fenômeno de Gibbs requeria o uso de filtros a fim de produzir resultados precisos. A eliminação das oscilações espúrias, associadas ao fenômeno de Gibbs presente em séries de Fourier, expandiram consideravelmente sua aplicabilidade. Um exemplo concreto disso, no âmbito dos problemas a valores no contorno, está presente na solução de problemas envolvendo condições de contorno não-periódicas, pois nesses casos o emprego de séries de Fourier clássicas não era recomendado (ver, por exemplo, [5], [2], [1]).

\subsection{Resolução da Eq. de Poisson por FG}

Consideremos, inicialmente, a equação de Poisson unidimensional $-u^{\prime \prime}=f$ sobre I com condições de contorno homogêneas e, por exemplo, $f=\exp \left(x^{2}\right)\left(2+4 x^{2}\right)$. 


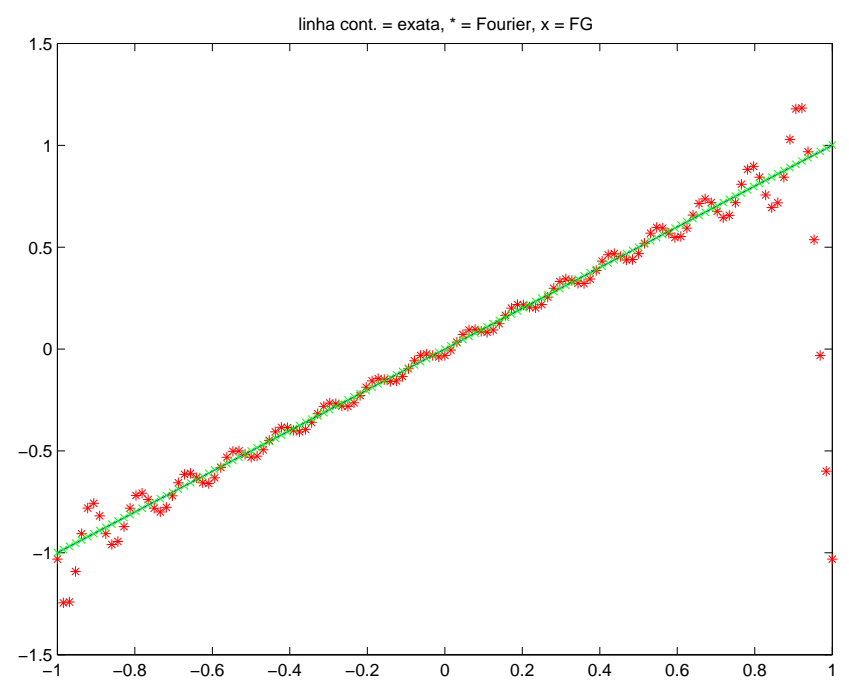

Figura 1: Aproximação de $f(x)=x$ por Fourier e por Fourier-Gegenbauer, com $\mathrm{N}=32$ e $\alpha=\beta=1 / 4$.

Pelo método de Fourier, podemos facilmente obter uma aproximação para os coeficientes de Fourier de $\mathrm{u}, \widehat{u}$ :

$$
\widehat{u}_{k}=\frac{\widehat{f}_{k}+\widehat{f}_{0}(-1)^{k+1}}{k^{2} \pi^{2}}, 0<|k| \leq N .
$$

Uma vez que $\widehat{u}_{0}=-\sum(-1)^{k} \widehat{u}_{k}$ converge lentamente (devido ao termo envolvendo o modo fundamental $\left.\widehat{f}_{0}\right), \widehat{u}_{0}$ é aproximado através da sequinte expressão:

$$
\widehat{u}_{0} \cong \frac{2 \widehat{f}_{0}}{\pi^{2}} \sum_{k=1}^{N} \frac{1}{k^{2}}+\frac{1}{\pi^{2}} \sum_{0<|k| \leq N} \frac{(-1)^{k+1}}{k^{2}} \widehat{f}_{k} \cong \frac{\widehat{f}_{0}}{3}+\frac{1}{\pi^{2}} \sum_{0<|k| \leq N} \frac{(-1)^{k+1}}{k^{2}} \widehat{f}_{k}
$$

Tabela 1: Erro máximo entre as aproximações de Fourier e Fourier-Gegenbauer em função de N. Equação de Poisson 1-D com $f=2$.

\begin{tabular}{|l|l|l|}
\hline $\mathrm{N}$ & $\left\|E_{F o}\right\|_{\infty}$ & $\left\|E_{F G}\right\|_{\infty}$ \\
\hline 4 & $8.9 \mathrm{E}-2$ & $7.5 \mathrm{E}-1$ \\
\hline 8 & $4.7 \mathrm{E}-2$ & $4.1 \mathrm{E}-4$ \\
\hline 16 & $2.4 \mathrm{E}-2$ & $1.8 \mathrm{E}-5$ \\
\hline 32 & $1.2 \mathrm{E}-2$ & $9.3 \mathrm{E}-8$ \\
\hline 64 & $6.3 \mathrm{E}-3$ & $6.6 \mathrm{E}-7$ \\
\hline 128 & $3.2 \mathrm{E}-3$ & $8.5 \mathrm{E}-5\left(^{*}\right)$ \\
\hline
\end{tabular}


A representação em série de Fourier resultante $\sum_{|k| \leq N} \hat{u}_{k} e^{i k \pi x}$ é afetada pela não-periodicidade da solução e exibe taxa de convergência algébrica (uma vez que $\widehat{u}_{k} \sim k^{-2}$ ). Na Tabela 1, mostra-se o resultado do pós-processamento destes coeficientes via equação (2.4), $\alpha=\beta=1 / 4$ e $f=2$. Como conseqüência da equação (2.5), a re-expansão em polinômios de Gegenbauer $u_{N}^{F G}(x)=\sum_{k=0}^{m} d_{k}^{\lambda} C_{k}^{\lambda}(x)$ com $d_{k}^{\lambda}$ obtido da equação (2.4) em termos dos coeficients de Fourier, produz uma aproximação com acurácia espectral. No entanto, observa-se que para $\mathrm{N}=128$, os erros de arredondamento crescem rapidamente. Na Tabela 2, com $f=\exp \left(x^{2}\right)\left(2+4 x^{2}\right)$ (solução exata $u=\exp (1)-\exp \left(x^{2}\right)$ ), observa-se uma taxa de convergência mais lenta e uma influência mais intensa dos erros de arredondamento para $N \geq 128$. Em ambas as tabelas, os resultados indicados por $\left(^{*}\right)$ foram obtidos com quadraduras de Gauss-Legendre (para obter coeficientes de Fourier mais exatos que os fornecidos pela FFT, e assim permitir uma avaliação mais precisa dos somatórios). Para valores maiores de $\mathrm{N}$, os resultados de FG não fazem sentido, a menos que correções adequadas sejam introduzidas. A causa da convergência lenta neste caso é a presença de fortes gradientes próximo aos contornos, que geram erros de regularização elevados.

Observa-se que no caso de soluções suaves, atinge-se uma aproximação adequada antes (pequenos valores de N) que os efeitos dos erros de arredondamento interfiram significativamente na qualidade da aproximação. Tais erros estão associados a: (i) avaliações de somatórios (com termos envolvendo sinais alternados), (ii) aproximações das funções Gama e de Bessel (ver equação (2.4)), (iii) ao uso da transformada rápida de Fourier para funções não-periódicas.

Soluções $C^{\infty}$ com singularidades próximas à origem (ver [3], pg. 90; os polinômios de Gegenbauer tem propriedade similar), fortes gradientes ou comportamento altamente oscilatório requerem muitos termos na expansão em polinômios de Gegenbauer para atingir resultados de elevada acurácia. Em [13], uma suavização preliminar (denominada subtração polinomial por [5], p. 77) foi empregada para melhorar a convergência das aproximações de FG de funções altamente oscilatórias. Um procedimento para lidar com soluções envolvendo fortes gradientes nos contornos do domínio é apresentado em [12]. Observamos também que técnicas de subtração de singularidades (ver, por exemplo, [1]) também podem ser implementadas para reduzir erros de regularização e acelerar a convergência das expansões de Gegenbauer.

Tabela 2: Erro máximo entre as aproximações de Fourier e Fourier-Gegenbauer como uma função de N. Equação de Poisson 1-D com $f=\exp \left(x^{2}\right)\left(2+4 x^{2}\right)$.

\begin{tabular}{|l|l|l|}
\hline $\mathrm{N}$ & $\left\|E_{F o}\right\|_{\infty}$ & $\left\|E_{F G}\right\|_{\infty}$ \\
\hline 4 & $2.4 \mathrm{E}-1$ & 1.3 \\
\hline 8 & $1.3 \mathrm{E}-1$ & $3.6 \mathrm{E}-1$ \\
\hline 16 & $6.7 \mathrm{E}-2$ & $8.3 \mathrm{E}-2$ \\
\hline 32 & $3.3 \mathrm{E}-2$ & $2.0 \mathrm{E}-3$ \\
\hline 64 & $1.7 \mathrm{E}-2$ & $2.8 \mathrm{E}-6$ \\
\hline 128 & $8.6 \mathrm{E}-3$ & $2.2 \mathrm{E}-4\left(^{*}\right)$ \\
\hline
\end{tabular}




\subsection{Resolução da Equação de Helmholtz por FG}

No caso da equação de Helmholtz, $-u^{\prime \prime}+\mu^{2} u=f$ para $x \in I$, com condições de contorno de Dirichlet homogêneas, o procedimento direto falha conforme descrevemos a seguir. A transformada finita de Fourier desta equação é dada por

$$
\widehat{u}_{k}=\frac{\widehat{f}_{k}-\mu_{0}(-1)^{k}}{k^{2} \pi^{2}+\mu^{2}}
$$

onde $\mu_{0}:=\left[u^{\prime}(1)-u^{\prime}(-1)\right] / 2$. O coeficiente associado ao modo fundamental é obtido por integração da equação diferencial:

$$
\widehat{u}_{0}=\frac{\widehat{f}_{0}-\mu_{0}}{\mu^{2}} .
$$

Finalmente, a terceira equação (os coeficientes de Fourier $\widehat{u}_{k}$, o coeficiente fundamental $\widehat{u}_{0}$ e $\mu_{0}$ são incógnitas) é obtida ao impormos as condições de contorno:

$$
\widehat{u}_{0}=-\sum_{0<|k| \leq N} \widehat{u}_{k}(-1)^{k} .
$$

Após resolver estas três equações, obtemos expressões para as aproximações $u_{N}$ que não convergem espectralmente à solução exata (denominemos este caso helma) quando $N \rightarrow \infty$. Isso ocorre porque as expressões resultantes contêm somas que convergem lentamente, e sem uma representação exata (como no caso anterior).

Proposição 3.1. Sejam $f \in L^{2}(I)$ e $u \in H_{0}^{1}(I) \cap H^{2}(I)$ tais que $-u^{\prime \prime}+\mu^{2} u=$ $f, x \in I$, então:

$$
\omega_{1} \hat{u}_{0}=\hat{f}_{0} \omega_{2}+\frac{3}{\pi^{2}} \sum_{0<|k|<\infty}(-1)^{k+1}\left(1-\frac{\mu^{2}}{\mu^{2}+k^{2} \pi^{2}}\right) \frac{\hat{f}_{k}}{k^{2}},
$$

onde

$$
\omega_{1}=3+\mu^{2} \omega_{2}, \quad \omega_{2}=1-\frac{3}{\pi^{2}} \sum_{0<|k| \leq \infty} \frac{\mu^{2}}{\left(\mu^{2}+k^{2} \pi^{2}\right) k^{2}} .
$$

Além disso,

$$
\widehat{u}_{k}=\frac{\widehat{f}_{k}+(-1)^{k}\left[\mu^{2} \hat{u}_{0}-\hat{f}_{0}\right]}{k^{2} \pi^{2}+\mu^{2}} .
$$

Demonstração. Substituindo o novo forçante $f-\mu^{2} u$ na versão exata (sem truncamento das séries) da equação (3.2), obtém-se:

$$
\widehat{u}_{0}=\frac{\widehat{f}_{0}-\mu^{2} \widehat{u}_{0}}{3}+\frac{1}{\pi^{2}} \sum_{0<|k|<\infty}(-1)^{k+1} \frac{\widehat{f}_{k}-\mu^{2} \widehat{u}_{k}}{k^{2}} .
$$


Procedendo de forma similar com relação a equação (3.1), obtem-se:

$$
\widehat{u}_{k}=\frac{\widehat{f}_{k}-\mu^{2} \widehat{u}_{k}+\left(\widehat{f}_{0}-\mu^{2} \widehat{u}_{0}\right)(-1)^{k+1}}{k^{2} \pi^{2}}, 0<|k|<\infty,
$$

ou seja,

$$
\widehat{u}_{k}=\frac{\widehat{f}_{k}+\left(\mu^{2} \widehat{u}_{0}-\widehat{f}_{0}\right)(-1)^{k}}{k^{2} \pi^{2}+\mu^{2}}, 0<|k|<\infty .
$$

Substituindo esta última expressão na equação (3.9) e efetuando-se algumas manipulações algébricas, obtém-se a expressão apresentada no enunciado da proposição.

Tabela 3: Comparação entre os erros máximos obtidos nos casos helma e helmb. Ambos os casos resolvem a equação de Helmholtz com condições de contorno homogêneas de Dirichlet e $f=\left(17-18 x^{2}+x^{4}\right) / 12$. Incluimos, também, o resultado do método de Fourier (em helmb) sem pós-processar os coeficientes.

\begin{tabular}{|c|c|c|c|}
\hline $\mathrm{N}$ & $\|e\|_{\infty}^{\text {helmb }}$ & $\|$ e $\|_{\infty}^{\text {helma }}$ & $\|e\|_{\infty}^{\text {Fob }}$ \\
\hline 8 & $3.0 \mathrm{E}-1$ & $2.8 \mathrm{E}-1$ & $3.0 \mathrm{E}-2$ \\
\hline 16 & $3.7 \mathrm{E}-2$ & $5.3 \mathrm{E}-2$ & $1.6 \mathrm{E}-2$ \\
\hline 32 & $3.0 \mathrm{E}-6$ & $1.1 \mathrm{E}-2$ & $8.2 \mathrm{E}-3$ \\
\hline 64 & $3.7 \mathrm{E}-7$ & $4.2 \mathrm{E}-3$ & $4.2 \mathrm{E}-3$ \\
\hline 128 & $5.1 \mathrm{E}-8$ & $2.1 \mathrm{E}-3$ & $2.1 \mathrm{E}-3$ \\
\hline
\end{tabular}

Empregando a proposição apresentada, recupera-se a acurácia desejada para a aproximação $u_{N}$ (caso helmb - Tabela 3). Naturalmente, se $\mu=0$, recupera-se o resultado obtido no caso anterior (equação de Poisson 1D), equação (3.2).

Em [13], os autores encontraram dificuldades na resolução da equação de Helmholtz com condição de contorno $-\mathrm{u}(-1)=\mathrm{u}(1)=1$ e valores elevados para $\mu$.

Consideremos novamente a função $u=\exp (1)-\exp \left(x^{2}\right)$ como a solução exata da equação de Helmholtz com condições de contorno homogêneas de Dirichlet. Neste caso, $f(x)=\exp \left(x^{2}\right)\left(2+4 x^{2}\right)+\mu^{2} u(x)$.

Descrevemos um método proposto em [13] a fim de compará-lo com nosso método. Os autores aplicaram a transformada finita de Fourier à equação de Helmholtz sem considerar as condições de contorno

$$
\hat{u}_{k}=\frac{\hat{f}_{k}}{k^{2} \pi^{2}+\mu^{2}}(k=-N,-N+1, \ldots, N)
$$

(com a equação de Helmholtz na forma $-u^{\prime \prime}+\mu^{2} u=f,-1<x<1$ ). A solução particular $u_{p}$ não satisfaz às condições de contorno. Então, faz-se a seguinte correção:

$$
u(x)=u_{p}(x)+h_{1}(x)+h_{2}(x)
$$


onde $h_{1}(x)=\alpha \exp (\mu x)$ e $h_{2}(x)=\beta \exp (-\mu x)$. Os coeficientes $\alpha$ e $\beta$ são determinados pelas condições de contorno

$$
\begin{aligned}
& h_{1}(-1)+h_{2}(-1)=-u_{p}(-1), \\
& h_{1}(1)+h_{2}(1)=-u_{p}(1) .
\end{aligned}
$$

A solução particular $u_{p}$ é obtida a partir de uma expansão em polinômios de Gegenbauer, onde os coeficientes da expansão são obtidos a partir dos coeficientes de Fourier $\hat{u}_{k} \cdot u_{p}( \pm 1)$ é avaliado através da expansão em polinômios de Gegenbauer nos pontos \pm 1 . $G(\exp ( \pm \mu x))$ denota a aproximação de Fourier-Gegenbauer das funções $\exp (\mu x)$ e $\exp (-\mu x)$.

Tabela 4: Erro máximo para vários valores de $\mathrm{N}$ com $\alpha=\beta=0.25$ e $\mu=80$ : FG (método proposto por VWI, [13]), método FG1 proposto neste trabalho (sem empregar a Proposição 3.1) combinado com a técnica proposta por VWI, e método FG2 proposto neste trabalho (empregando a Proposição 3.1). $u=\exp (1)-\exp \left(x^{2}\right)$ como a solução exata da equação de Helmholtz com condições de contorno homogêneas de Dirichlet. Neste caso, $f(x)=\exp \left(x^{2}\right)\left(2+4 x^{2}\right)+\mu^{2} u(x)$.

\begin{tabular}{|c|c|c|c|}
\hline $\mathrm{N}$ & $\|e\|_{\infty}^{V W I}$ & $\|e\|_{\infty}^{F G 1}$ & $\|e\|_{\infty}^{F G 2}$ \\
\hline 4 & 1.3 & 1.3 & 1.3 \\
\hline 8 & $3.6 \mathrm{E}-1$ & $3.6 \mathrm{E}-1$ & $3.6 \mathrm{E}-1$ \\
\hline 16 & $8.3 \mathrm{E}-2$ & $8.3 \mathrm{E}-2$ & $8.3 \mathrm{E}-2$ \\
\hline 32 & $2.0 \mathrm{E}-3$ & $2.0 \mathrm{E}-3$ & $2.0 \mathrm{E}-3$ \\
\hline 64 & $1.5 \mathrm{E}-5$ & $7.6 \mathrm{E}-6$ & $1.8 \mathrm{E}-6$ \\
\hline
\end{tabular}

Na Tabela 4, apresentamos os resultados do método de VWI ([13]) aplicado ao nosso problema. O desempenho da técnica não é tão bom como no caso-teste mais simples (com $\mathrm{u}=\mathrm{x}$ ) descrito naquele trabalho ([13]). Nesta tabela adicionamos dois outros casos: nosso método de FG sem uso da Proposição 3.1, e com o uso daquela proposição. As performances são distintas apenas em $\mathrm{N}=128$.

Comparando o método de FG proposto (baseado na Proposição 3.1) com a técnica de VWI ([13]) para diferentes valores de $\mu$ (Tabela 5), observa-se que a técnica de VWI apresenta um comportamento estranho em $\mu=20$ com um crescimento e posterior decaimento do erro. Uma vez que o custo computacional é dominado pelo cálculo dos coeficientes de Gegenbauer a partir dos coeficientes de Fourier, e a técnica VWI emprega dois processamentos de tais coeficientes, e exibe perda de acurácia para certos valores de $\mu$, conclui-se que o método de FG proposto neste trabalho é vantajoso nestes aspectos. Isso é esperado uma vez que levamos em conta os valores de contorno nas equações transformadas, o que torna desnecessário efetuar correções subseqüentes.

Observação: Na prática, verificamos a convergência para a solução exata (desconhecida, em geral) da seguinte forma:

(i) avaliamos a expansão em polinômios de Gegenbauer nos pontos de colocação de 


\section{Chebyshev;}

(ii) avaliamos a expansão em série de Chebyshev que interpola os valores dados nos pontos de colocação;

(iii) através de subrotinas que realizam diferenciação numérica (Chebyshev), avaliamos o resíduo da equação (Lu - f) nos pontos de colocação;

(iv) se a norma do resíduo é menor que uma dada tolerância $\epsilon$, então, considera-se a solução obtida como satisfatória.

O custo computacional adicional envolvido nesta avaliação está entre 2 e $4 \%$ do custo total. Este procedimento tem importância prática, pois aproximações contaminadas por erros de arredondamento (observadas em exemplos anteriores) são facilmente identificadas pelos elevados valores do resíduo calculado neste pósprocessamento.

Como um exemplo, o resíduo na resolução aproximada da equação de Helmholtz unidimensional para $\mu=80$ com o método proposto e $u_{\text {exact }}(x)=e^{1-x^{2}}-1$ tem valor máximo igual a $8.0 E-4$ (o erro máximo é igual a $1.4 E-7$ ) para $N=64$.

Tabela 5: Erro máximo no método de FG proposto com uso da Proposição $3.1 \mathrm{e}$ no método de FG (técnica de VWI), em termos de N com $\alpha=\beta=0.25$ e $N=64$. $u=\exp (1)-\exp \left(x^{2}\right)$ como a solução exata da equação de Helmholtz com condições de contorno homogêneas de Dirichlet. Neste caso, $f(x)=\exp \left(x^{2}\right)\left(2+4 x^{2}\right)+$ $\mu^{2} u(x)$.

\begin{tabular}{|c|c|c|}
\hline$\mu$ & $\|e\|_{\infty}^{F G}$ & $\|e\|_{\infty}^{V W T}$ \\
\hline 1 & $2.3 \mathrm{E}-6$ & $1.5 \mathrm{E}-7$ \\
\hline 20 & $4.5 \mathrm{E}-5$ & $4.3 \mathrm{E}-2$ \\
\hline 40 & $2.0 \mathrm{E}-5$ & $1.0 \mathrm{E}-2$ \\
\hline 60 & $3.8 \mathrm{E}-6$ & $3.8 \mathrm{E}-4$ \\
\hline 80 & $1.8 \mathrm{E}-6$ & $1.5 \mathrm{E}-5$ \\
\hline 100 & $1.6 \mathrm{E}-6$ & $3.8 \mathrm{E}-7$ \\
\hline 200 & $1.6 \mathrm{E}-6$ & $1.6 \mathrm{E}-6$ \\
\hline
\end{tabular}

\section{Exemplo Adicional}

O último caso ilustrativo baseia-se na seguinte equação hiperbólica, que abordaremos com a técnica proposta (diferentemente de [5]),

$$
\begin{aligned}
\frac{\partial u}{\partial t}+\frac{\partial u}{\partial x} & =x+t, 0<x<\pi, t>0 \\
u(0, t) & =0, t \geq 0 \\
u(x, 0) & =0,0 \leq x \leq \pi
\end{aligned}
$$

A solução exata deste problema é $u=x t$. Novamente, violamos a regra tradicional que recomenda o uso de expansões em série em termos de funções não-periódicas 
(por exemplo, polinômios de Chebyshev/Legendre). Representamos u com uma série de Fourier truncada.

$$
u_{N}=\sum_{k=-N}^{N} \hat{u}_{k}(t) e^{i k x} .
$$

Então, obtemos o conjunto usual de EDO's para os coeficientes de Fourier:

$$
\begin{gathered}
\widehat{u}_{k}^{\prime}=-i k \widehat{u}_{k}+(\widehat{x+t})_{k}, 0<|k| \leq N, \\
\widehat{u}_{k}(0)=0,
\end{gathered}
$$

onde o modo fundamental $\widehat{u}_{o}$ é dado por $\widehat{u}_{o}=t^{2} / 2$ (obtido naturalmente por integração direta da equação diferencial). Expressões matemáticas alternativas para o cálculo deste coeficiente (por exemplo, $\widehat{u}_{0}=\sum \frac{\widehat{u}_{k}^{\prime}-(\widehat{x+t})_{k}}{i k}$ ) convergem lentamente e introduzem erros de arredondamento que impossibilitam a obtenção da acurácia espectral a partir da série polinomial produzida pelo método. A Tabela 6 exibe a comparação entre as aproximações de Fourier e Fourier-Gegenbauer.

Tabela 6: Erro máximo da expansão de Fourier e da expansão de Gegenbauer, como uma função do número de modos de Fourier $\left(\alpha_{x}=\alpha_{y}=0.25\right)$ para a equação hiperbólica.

\begin{tabular}{|c|c|c|c|c|}
\hline $\mathrm{N}$ & 4 & 8 & 16 & 32 \\
\hline$\|e\|_{\infty}^{G}$ & $1.899 \mathrm{E}-3$ & $8.850 \mathrm{E}-5$ & $4.3506 \mathrm{E}-7$ & $1.757 \mathrm{E}-11$ \\
\hline$\|e\|_{\infty}^{F}$ & $1.827 \mathrm{E}-1$ & $9.835 \mathrm{E}-2$ & $3.636 \mathrm{E}-2$ & $2.100 \mathrm{E}-2$ \\
\hline
\end{tabular}

Este exemplo ilustra o seguinte fato: dentre todos os coeficientes de Fourier obtidos aproximadamente (via FFT), o coeficiente associado ao modo fundamental (que nesse caso pôde ser avaliado exatamente) é aquele que tem impacto maior sobre a acurácia final da aproximação obtida pelo método de Fourier-Gegenbauer.

\section{Sumário}

Propusemos um esquema numérico de resolução das equações de Helmholtz baseado no método de Fourier-Gegenbauer, originalmente proposto em [6]. Obtivemos acurácia espectral para dados suaves (analíticos ou $C^{\infty}$ com expansões em polinômios de Gegenbauer rapidamente convergentes) ao pós-processar os coeficientes de Fourier a fim de remover as oscilações espúrias associadas ao fenômeno de Gibbs. O estudo de casos e comparações com outras técnicas na literatura ilustrou a aplicabilidade do método bem como algumas dificuldades originadas pela rápida propagação dos erros de arredondamento. No trabalho, obtivemos um procedimento simples para resolver as equações de Poisson e Helmholtz unidimensionais sem condições de contorno periódicas. Comparações com técnicas apresentadas na literatura mostraram melhores resultados para o método proposto neste trabalho, nos casos-teste considerados. 
Abstract. Gottlieb et al. proposed in [6] a new approach to overcome the Gibbs phenomenon of Fourier series expansions of discontinuous, piecewise analytic functions. Their approach uses the Fourier coefficients to obtain the coefficients of a Gegenbauer expansion used to represent with spectral accuracy the given function. In this paper, we propose a one-dimensional Fourier-Gegenbauer Helmholtz solver of high accuracy. Numerical case studies and comparisons with other approaches in the literature show improved results.

\section{Agradecimentos}

Os autores agradecem aos revisores, que contribuiram em vários aspectos para tornar o texto mais claro e correto.

\section{Referências}

[1] J.P. Boyd, "Chebyshev and Fourier Spectral Methods", 2nd. Ed., Dover, New York, 2000.

[2] C. Canuto, M.Y. Hussaini, A. Quarteroni e T.A. Zang, "Spectral Methods in Fluid Dynamics", Springer-Verlag, 1988.

[3] P.J. Davis, "Interpolation and Approximation", Dover, 1975.

[4] A. Gelb e D. Gottlieb, The resolution of the Gibbs phenomenon for "spliced" functions in one and two dimensions, Computers Math. Applic., 33, No. 11 (1997), 35-58.

[5] D. Gottlieb e S.A. Orszag, "Numerical Analysis of Spectral Methods: Theory and Applications", SIAM-CBMS, 1977.

[6] D. Gottlieb, C.-H. Shu, A. Solomonoff e H. Vandeven, On the Gibbs Phenomenon I: Recovering Exponential Accuracy from the Fourier Partial Sum of a Non-periodic Analytic Function, J. Comput. Appl. Math., 43 (1992), 81-98.

[7] D. Gottlieb e C.-H. Shu, Resolution Properties of the Fourier Method for Discontinuous Waves, Comput. Meth. Appl. Mech. Engin., 116 (1994), 27-37.

[8] D. Gottlieb e C.-H. Shu, On the Gibbs Phenomenon III: Recovering Exponential Accuracy in a Sub-interval from the Spectral Partial Sum of a Piecewise Analytic Function, SIAM J. Numer. Anal., 33 (1996), 280-290.

[9] D. Gottlieb e C.-H. Shu, On the Gibbs Phenomenon IV: Recovering Exponential Accuracy in a Sub-interval from the Gegenbauer Partial Sum of a Piecewise Analytic Function, Math. Comp., 64 (1995), 1081-1095.

[10] D. Gottlieb e C.-H. Shu, On the Gibbs Phenomenon V: Recovering Exponential Accuracy from Collocation Point Values of a Piecewise Analytic Function, Numer. Math., 71 (1995), 511-526.

[11] D. Gottlieb e C.-H. Shu, On the Gibbs Phenomenon and Its Resolution, SIAM Review, 39, No. 4 (1997), 644-668. 
[12] L. Vozovoi, M. Israeli e A. Averbuch, Analysis and Application of FourierGegenbauer Method to Stiff Differential Equations, SIAM J. Numer. Anal., 33 (1996), 1844-1863.

[13] L. Vozovoi, A. Weill e M. Israeli, Spectrally Accurate Solution of Non-periodic Differential Equations by the Fourier-Gegenbauer Method, SIAM J. Numer. Anal., 34 (1997), 1451-1471. 